\title{
Axiomatisability of the Class of Monolithic Groups in a Variety of Nilpotent Groups
}

\author{
Joshua T. Grice
}

November 27, 2019

\begin{abstract}
The class of all subdirectly irreducible groups belonging to a variety generated by a finite nilpotent group can be axiomatised by a finite set of elementary sentences.
\end{abstract}

\section{Introduction}

A group is subdirectly irreducible or monolithic if it has some minimal nontrivial normal subgroup, called its monolith. A variety of groups is a class of groups closed with respect to homomorphic images, subgroups, and arbitrary direct products. According to a fundamental result of Garrett Birkhoff in his 1935 paper 2], a variety is also a proper class of groups that is axiomatised by some set of equations. Given a group $\mathbf{G}$, the variety generated by $\mathbf{G}$, denoted $\mathcal{V}(\mathbf{G})$, is the smallest variety to which $\mathbf{G}$ belongs, or equivalently the intersection of all varieties containing $\mathbf{G}$.

By another theorem of Birkhoff's in a 1944 paper [3], two varieties are equal if they share the same subdirectly irreducible members. Thus, given a variety $\mathcal{V}$, the class of its subdirectly irreducible members, commonly denoted $\mathcal{V}_{\mathrm{si}}$, is of particular interest. Subdirect irreduciblity as a property is not preserved by the formation of direct products, and so $\mathcal{V}_{\mathrm{si}}$ is not a variety and cannot be determined by a finite set of equations. But it can still be axiomatised by a finite set of elementary sentences, which are more general first-order statements.

The main result of this paper is as follows.

Theorem 1.1. Let $\mathcal{V}$ be a variety generated by a finite nilpotent group $\mathbf{G}$. Then, the class of subdirectly irreducible groups belonging to $\mathcal{V}$ is axiomatisable by a finite set of elementary sentences.

Finite axiomatisability of various structures has been studied at length by logicians, abstract algebraists and universal algebraists. In the realm of groups, Lyndon proved in 1952 6 that the variety generated by any nilpotent group is finitely axiomatisable. In 1965, Oates and Powell 8 proved that the variety generated by any finite group is finitely axiomatisable. In both of these cases, the axioms can be taken to be equations.

A broader result than our main theorem was a hair's breadth from being proved by George F. McNulty and Wang $\mathrm{Ju}$ in 2000. A preprint was circulated in which it was claimed that the subdirectly irreducible members of a variety generated by any finite group was finitely axiomatisable. However, an error in the proof caused much of the work to be unusable. The current paper is an attempt to salvage some of that work, and has managed to prove the assertion with the added 
hypothesis of nilpotence. The author wishes to thank Dr. McNulty personally for his devoted guidance as a $\mathrm{PhD}$ advisor, and for this problem in particular.

Baker and Wang proved in 2002 in [1] that for certain kinds of varieties, $\mathcal{V}$ itself being finitely axiomatisable and $\mathcal{V}_{\mathrm{si}}$ being finitely axiomatisable are in fact equivalent. It is that result, and the aforementioned importance of subdirectly irreducible members of varieties, that motivates investigation into finite axiomatisability of subdirectly irreducible algebras.

\section{Preliminaries}

\subsection{Elementary Logic}

Our result dwells in first-order, or elementary logic, using the language of groups. The terms of the language of groups are built up from variables representing elements of the group joined together with the multiplication and inverse operations of groups, and the named constant symbol 1 representing the identity. For instance, the conjugate $x y x^{-1}$ is a term in this language. The variables cannot be used to represent sequences or subgroups, only elements; hence the name elementary logic.

An equation is some statement of equality between two terms whose variables are understood to range over the whole group. For instance, the commutative law of Abelian groups can be expressed as an equation:

$$
x y \approx y x
$$

An elementary formula is built up from equations in a systematic way with the help of logical connectives $\vee, \wedge, \neg, \leftarrow$, and $\leftrightarrow$ (conjunction, disjunction, negation, implication and biconditional, respectively), and the quantifiers $\exists$ and $\forall$. A formula may look something like:

$$
\forall y(x y \approx y x) \wedge \neg(x \approx 1)
$$

Note that in this formula, the variable $x$ appears but is not quantified. This makes $x$ a free variable, and illustrates how formulas can be used to define sets of elements. If the above formula is named $\Phi$, for instance, the set defined by $\Phi(x)$ would be the set of all elements $x$ of a group that satisfy that formula. In this case, $\Phi(x)$ is the set of nontrivial elements of the group's center.

If a formula has no free variables, it is called an elementary sentence. A sentence in the language of groups is either true or false in a given group, whereas a formula depends on the value that the variables take. Sentences are useful for stating laws obeyed in a structure that cannot be expressed by equations alone. For example, the presence of an inverse for every element of a group:

$$
\forall x \exists y(x y \approx 1)
$$

Let $\mathcal{K}$ be a proper class of structures. If $\Sigma$ is a finite set of sentences so that a given structure $\mathbf{A}$ belongs to $\mathcal{K}$ if and only if it satisfies every sentence in $\Sigma$, we say that $\mathcal{K}$ is finitely axiomatisable. When axiomatising varieties, we note that these sentences can all be taken to be equations.

\subsection{Group Theory}

We define the normal closure of a set $X$ of elements of a group $\mathbf{G}$ as the smallest normal subgroup containing $X$. We may also call it the normal subgroup generated by $X$. We denote this subgroup by $X^{G}$. If $X$ is a singleton set, say $X=\{a\}$, we call this subgroup a principal normal subgroup and 
write $a^{G}$. Note that in a subdirectly irreducible group, the monolith cannot contain any nontrivial normal subgroup and is therefore always principal.

Given two elements $a, b$ of a given group $\mathbf{G}$, their commutator $[a, b]$ is the element $a b a^{-1} b^{-1}$. The commutator operation can be extended to normal subgroups; the commutator of two normal subgroups $\mathbf{H}$ and $\mathbf{K}$ of $\mathbf{G}$ is defined as $[\mathbf{H}, \mathbf{K}]=\{[h, k]: h \in H, k \in K\}$. The commutator of two normal subgroups is again a normal subgroup. Using the commutator operation, one may fabricate a lower central series $G_{0} \triangleright G_{1} \triangleright G_{2} \triangleright \ldots$ where $G_{0}=\mathbf{G}$ and $G_{i}=\left[G, G_{i}\right]$. The group $\mathbf{G}$ is called nilpotent of class $k$ if there is some $k$ for which $G_{k}=\{1\}$.

An equivalent (and, for our purposes, more useful) definition of nilpotence is the presence of an upper central series $Z_{0} \triangleleft Z_{1} \triangleleft Z_{2} \triangleleft \ldots$ so that $\{1\}=Z_{0}$ and, for each $i, Z_{i+1} / Z_{i}=Z\left(\mathbf{G} / Z_{i}\right)$. The group $\mathbf{G}$ is nilpotent if there is some $k$ for which $Z_{k}=\mathbf{G}$. It is well-known that the length of the upper and lower central series coincides; a proof can be found in Dummit and Foote [4. A third well-known characterisation of nilpotence exists; a group is nilpotent if and only if it is the direct product of its Sylow subgroups.

By Lyndon's work, the nilpotence class of a group can be captured with a finite set of equations [6. Therefore, if $\mathbf{G}$ is nilpotent of class $k$, any group $\mathbf{H} \in \mathcal{V}(\mathbf{G})$ is nilpotent of class at most $k$.

Given a group $\mathbf{G}$, the normal subgroups of $\mathbf{G}$ form a lattice. If $K<H$ are normal subgroups of G and there exists no normal subgroup $N$ so that $H \leq N \leq K$, then $H / K$ is called a chief factor of G. According to Hanna Neumann's 1967 book [7, the cardinality of chief factors in a variety generated by a finite group $\mathbf{G}$ is bounded above by $|\mathbf{G}|$.

\section{Definable Principal Normal Subgroups}

Our proof of the finite axiomatisability of $\mathcal{V}_{\mathrm{si}}$ is contingent on a useful definition from Baker and Wang's 2002 paper [1] that they called definable principal subcongruences. Their formulation of this concept is from a perspective of general algebraic structures, so we will rework it here to focus purely through the spyglass of group theory.

Let $\Phi(x, y)$ be an elementary formula. We will say that $\Phi$ is a normal closure formula provided that for any group $\mathbf{H}$, if $\Phi(a, b)$ holds in $\mathbf{H}$, then $a$ belongs to the normal closure $b^{\mathbf{H}}$. For instance, the formula

$$
\exists z\left(x \approx z y z^{-1}\right)
$$

is a normal closure formula, since any conjugate of the element $b$ will belong to $b^{\mathbf{H}}$. Normal closure formulas are useful for capturing the principal normal subgroups of a given group or class of groups in a way that is compatible with first-order logic, which in turn can help to axiomatise the groups themselves. A class of groups might be highly compatible with such a capturing; for some classes of groups, there might be one normal closure formula $\Phi(x, y)$ that can define every principal normal subgroup of every group in the class. Baker and Wang's definition is not quite so strong as that, but is in many ways the next best thing.

We will say that a class $\mathcal{K}$ of groups has definable principal normal subgroups if and only if there are normal closure formulas $\Phi(x, y)$ and $\Psi(x, y)$ so that for every $\mathbf{H} \in \mathcal{K}$ and every nonidentity $b \in H$, there exists a nonidentity $a \in H$ so that

1. $\mathbf{H} \models \Psi(a, b)$ and

2. $\Phi(x, a)$ defines the normal closure of $a$. 
In other words, if $b$ is an arbitrary element of $\mathbf{H}$, then $\Psi$ can find some nonidentity $a \in b^{H}$ so that $a^{H}$ is definable by $\Phi$.

In their paper, Baker and Wang use this definition to prove another finite axiomatisability result. Their result applies to more general algebraic structures, but we express it in terms of groups.

Theorem (Baker, Wang). Let $\mathcal{V}$ be a variety of groups and suppose that $\mathcal{V}$ has definable principal normal subgroups. Then, $\mathcal{V}$ is finitely axiomatisable if and only if $\mathcal{V}_{\mathrm{si}}$ is finitely axiomatisable.

A variation on the proof of this theorem yields the following result, whose proof we reproduce from McNulty and Wang's unpublished work. Again, the theorem holds for more general structures, but we state and prove it in terms of groups.

Theorem 3.1. If $\mathcal{V}$ is a variety of groups and $\mathcal{V}_{\mathrm{si}}$ has definable principal normal subgroups, then $\mathcal{V}_{\mathrm{si}}$ is finitely axiomatisable relative to $\mathcal{V}$. In particular, if $\mathcal{V}$ is finitely axiomatisable, then $\mathcal{V}_{\mathrm{si}}$ is finitely axiomatisable.

Proof. Let $\Sigma$ be a finite set of elementary sentences which axiomatises $\mathcal{V}$, and let $\Phi(x, y)$ and $\Psi(x, y)$ be the formulas witnessing that $\mathcal{V}_{\mathrm{si}}$ has definable principal normal subgroups. Let $\Theta$ be the following set of sentences:

$$
\Sigma \cup\{\exists u[u \neq 1 \wedge \forall z(z \neq 1 \Rightarrow \exists x(\Phi(u, x) \wedge \Psi(x, z)))]\}
$$

We claim that $\Theta$ axiomatises $\mathcal{V}_{\text {si }}$.

On one hand, suppose $\mathbf{S} \in \mathcal{V}_{\mathrm{si}}$. Let $c$ be a generator of the monolith of $\mathbf{S}$. So, $c \neq 1$ and $c$ belongs to every nontrivial normal subgroup. Now, let $b \in S-\{1\}$. Because $V_{\mathrm{si}}$ has definable principal normal subgroups, there exists some nonidentity $a \in S$ so that $S \models \Psi(a, b)$ and $\Phi(x, a)$ defines the normal closure $a^{S}$. Since $c$ generates the monolith, however, $c \in a^{S}$ also, and so $S \models \Phi(c, a)$. So,

$$
\mathbf{S} \models \exists u[u \neq 1 \wedge \forall z(z \neq 1 \Rightarrow \exists x(\Phi(u, x) \wedge \Psi(x, z)))]
$$

Since $\mathbf{S}$ belongs to $\mathcal{V}, \mathbf{S} \models \Sigma$ also. Therefore, $\mathbf{S} \models \Theta$.

Now, suppose $\mathbf{S} \models \Theta$. Then, $\mathbf{S} \in \mathcal{V}$ since $\Sigma$ axiomatises $\mathcal{V}$. But also, since $\mathbf{S}$ believes the second part of $\Theta$ and since $\Phi$ and $\Psi$ are normal closure formulas, there exists $c \in S-\{1\}$ so that $c$ is contained within any other principal normal subgroup. In particular, the principal normal subgroup $c^{H}$ is contained within any other principal normal subgroup of $\mathbf{S}$ and so $\mathbf{S}$ is subdirectly irreducible.

Thus, in view of Theorem 3.1 and the Oates-Powell theorem, to prove our main result we need only prove the following.

Theorem 3.2. Let $\mathcal{V}$ be a variety generated by a finite nilpotent group $\mathbf{G}$. Then, $\mathcal{V}_{\mathrm{si}}$ has definable principal normal subgroups.

We now introduce some machinery that will let us quantify principal normal subgroup inclusion in a first-order way. The set of conjugate product terms in $x$ of a variety of groups is the smallest set $C$ of terms so that

- $1 \in C$

- If $t \in C$ and $y$ is a variable, then both $\left(y x y^{-1}\right) t$ and $\left(y x^{-1} y^{-1}\right) t$ belong to $C$. 
The defintion is apt; $C$ is the set of all terms made by taking products of conjugates of $x$ and $x^{-1}$. A sample member of $C$ might be

$$
t\left(x, y_{0}, y_{2}, y_{7}\right)=y_{0} x y_{0}^{-1} y_{2} x^{-1} y_{2}^{-1} y_{7} x y_{7}^{-1}
$$

A conjugate product polynomial is a unary polynomial $\pi(x)$ forged from some conjugate product term. We might write $\pi(x, \bar{y})$ if we wish to specify the parameters. So, for instance, in some group $\mathbf{H}$, we might choose members $c_{0}, c_{2}, c_{7} \in H$ and, from our prior example, obtain the following conjugate product polynomial

$$
\pi(x)=t\left(x, c_{0}, c_{2}, c_{7}\right)=c_{0} x c_{0}^{-1} c_{2} x^{-1} c_{2}^{-1} c_{7} x c_{7}^{-1}
$$

Conjugate product polynomials are a powerful tool in groups; they are capable of defining principal normal subgroups. The normal closure of an element $a$, for instance, is the collection of products of conjugates of $a$ and $a^{-1}$, which is precisely the outputs of the sets of conjugate product polynomials in $a$. This arms us with a method of defining principal normal subgroups with objects that are easily written in first-order logic.

We refer to a statement of the form $a \in c^{H}$ as a membership condition. Membership conditions are our main object of interest in trying to establish definable principal normal subgroups, and we now have technology in the form of conjugate product polynomials to witness them. Our strategy in the proof will be to show that these conditions can be witnessed with a limited number of variables. This will enable us to quantify the witnessing using a first-order statement. In this paper, the complexity of a conjugate product polynomial refers to the number of conjugates present in the product. Our previous example has complexity 3.

\section{Proving Theorem 3.2}

In order to show that $\mathcal{V}_{\mathrm{si}}$ has definable principal normal subgroups, as desired, we need two different normal closure formulas. The first, $\Psi(x, y)$, to seek out some definable principal normal subgroup of any given principal normal subgroup, and the second, $\Phi(x, y)$, to do the defining. We will prove the existence of $\Phi$ first, using a proof of McNulty and Wang that appears in their unpublished paper that they have kindly allowed to be presented here. By an atom we mean a nontrivial normal subgroup $N$ of $\mathbf{G}$ which does not properly contain any other nontrivial normal subgroups of $\mathbf{G}$.

Theorem 4.1. Let $\mathcal{V}$ be the variety generated by a finite group. Then, there is a normal closure formula $\Phi(x, y)$ such that for any $\mathbf{H} \in \mathcal{V}$ and every $c \in H$ such that $c^{H}$ is an atom in the lattice of normal subgroups of $\mathbf{H}$, it follows that $\Phi(x, c)$ defines $c^{H}$.

Proof. Let $r$ be a finite upper bound on the size of chief factors in algebras belonging to $\mathcal{V}$. Then, we claim that if $c^{H}$ is an atom for some $c \in \mathbf{H}$, then any membership condition of the form $a \in c^{H}$ can be witnessed by a conjugate product polynomial of complexity no more than $r$.

If $a \in c^{H}$, then $a=g_{0} g_{1} \cdots g_{n-1}$ where each $g_{i}$ is some conjugate of either $c$ or $c^{-1}$. If $n$ is chosen to be as small as possible,

$$
g_{0}, \quad g_{0} g_{1}, \quad g_{0} g_{1} g_{2}, \ldots, g_{0} g_{1} \cdots g_{n-1}
$$

are $n$ distinct elements of $c^{H}$. Now, since $c^{H}$ is an atom, $c^{H} /\{1\}$ is a chief factor. $\left|c^{H}\right|=\left|c^{H} /\{1\}\right| \leq$ $r$, so $n \leq r$. 
Now, let $T$ be the set of all conjugate product terms in the signature of $\mathcal{V}$ whose parameters are chosen from the distinct variables $u_{0}, \ldots, u_{r-1}$. Since there are only finitely many variables being used, $T$ is finite. Now, let $\Phi(x, y)$ be the sentence

$$
\exists u_{0}, \ldots, u_{r-1}\left[\bigvee_{t \in T} t(y, \bar{u}) \approx x\right]
$$

$\Phi(x, c)$ now defines $c^{H}$ whenever $c^{H}$ is an atom.

Theorem4.1 gives us a normal closure formula that can define any atoms in any group belonging to $\mathcal{V}$; in particular, for any group in $\mathcal{V}_{\mathrm{si}}$, this formula will always define the group's monolith. The second formula that we need, $\Psi(x, y)$, will come from the following theorem, which is the original work of this paper.

Theorem 4.2. Let $\mathcal{V}$ be a variety generated by a group $\mathbf{G}$ of finite exponent $m$ and nilpotence class $k$. Let $\mathbf{S} \in \mathcal{V}_{\mathrm{si}}$. Then, given any $a \in \mathbf{S}$, there is some $b$ belonging to the monolith of $\mathbf{S}$ so that the membership condition $b \in a^{S}$ is witnessed by a conjugate product polynomial of complexity bounded above in terms of the generating group $\mathbf{G}$.

Proof. Since $\mathbf{S} \in \mathcal{V}$, the exponent of $\mathbf{S}$ divides that of $\mathbf{G}$, as the equation $x^{m}=1$ holds throughout $\mathcal{V}$. We also know that the nilpotence class $k$ of $\mathbf{S}$ is bounded above by that of $\mathbf{G}$; we harmlessly assume it is $k$. Denote the upper central series of $\mathbf{S}$ as

$$
\{1\}=Z_{0} \triangleleft Z_{1} \triangleleft \ldots \triangleleft Z_{k}=\mathbf{S}
$$

Note that $Z_{1}$ is the center of $\mathbf{S}$, which contains the monolith $M$ of $\mathbf{S}$. Choose any arbitrary $a \in S$. If $a \in M$, then no more work is needed, so we can assume it is not. Label $a=a_{k}$; now, we will form a sequence of elements walking down the steps of the central series that form a chain of principal normal subgroups. Given $a_{i+1} \in Z_{i+1}$, we will seek out $a_{i}$ so that the following hold:

1. $a_{i} \in Z_{i}$

2. $a_{i} \neq 1$

3. $a_{i} \in a_{i+1}^{S}$ and this fact is witnessed by a conjugate product polynomial of complexity at most $m$.

We can certainly find $a_{i} \in Z_{i}$ so that $a_{i} \in a_{i+1}^{S}$; since $\mathbf{S}$ is subdirectly irreducible, any element of the monolith $M$ will suffice. We choose $a_{i}$ from all such possible nonidentity candidates in $Z_{i}$ so that the conjugate product polynomial $\pi_{i}$ that witnesses $\pi_{i}\left(a_{i+1}, \bar{c}\right)=a_{i}$ has minimal possible complexity, and claim that this satisfies our above three requirements. The first two are already satisfied, so we need only worry about the complexity of $\pi_{i}$.

$\pi_{i}$ takes the form $\pi_{i}(x, \bar{c})=c_{0} x^{ \pm 1} c_{0}^{-1} c_{1} x^{ \pm 1} c_{1}^{-1}, \ldots c_{n} x^{ \pm 1} c_{n}^{-1}$ for some $n$. The structure of this polynomial breaks down into two cases.

Case 1) There are both positive and negative conjugates present in $\pi_{i}$. So, $\pi_{i}$ contains, somewhere, a product of the form

$$
c_{j} x c_{j}^{-1} c_{j+1} x^{-1} c_{j+1}^{-1}
$$

(or perhaps the same product with the negative conjugate on the left). We claim that these two conjugates are the whole of $\pi_{i}$, and that the element $c_{j} a_{i+1} c_{j}^{-1} c_{j+1} a_{i+1}^{-1} c_{j+1}^{-1}$, which we will 
temporarily call $a_{i}^{*}$, is in fact $a_{i}$ itself. Indeed, $a_{i}^{*}$ cannot be 1 ; if it were, these two conjugates could be removed from $\pi_{i}$ to preserve the given membership condition with a shorter polynomial, contradicting $\pi_{i}$ 's minimality. Clearly, $a_{i}^{*} \in a_{i+1}^{S}$. So all we need to do is show that $a_{i}^{*} \in Z_{i}$, and then the minimal complexity of $\pi_{i}$ will do the rest of the work for us.

Now, $Z_{i+1} / Z_{i}$ is the center of $\mathbf{S} / Z_{i}$, so $a_{i+1} / Z_{i}$ commutes with every member of $\mathbf{S} / Z_{i}$. So, we have

$$
\begin{aligned}
a_{i}^{*} / Z_{i} & =\left(c_{j} a_{i+1} c_{j}^{-1} c_{j+1} a_{i+1}^{-1} c_{j+1}^{-1}\right) / Z_{i} \\
& =\left(c_{j} / Z_{i}\right)\left(a_{i+1} / Z_{i}\right)\left(c_{j}^{-1} / Z_{i}\right)\left(c_{j+1} / Z_{i}\right)\left(a_{i+1}^{-1} / Z_{i}\right)\left(c_{j+1}^{-1} / Z_{i}\right) \\
& =\left(a_{i+1} / Z_{i}\right)\left(a_{i+1}^{-1} / Z_{i}\right) \\
& =1 / Z_{i}
\end{aligned}
$$

So, $a_{i}^{*} \in Z_{i}$. So, $a_{i}^{*}=a_{i}$, and the complexity of the polynomial needed to witness the membership $a_{i} \in a_{i+1}^{S}$ is 2 , which is certainly less than the exponent $m$ of $\mathbf{G}$ unless the variety is trivial.

Case 2) The conjugates present in $\pi_{i}$ are either all positive or all negative. We assume that the conjugates are all positive; if they are all negative, the proof is almost identical. In this case, we claim that the complexity of $\pi_{i}$ is at most $m$. The argument is similar to case 1 . Suppose the complexity is at least $m$; then, look at $a_{i}^{*}=c_{0} a_{i+1} c_{0}^{-1} c_{1} a_{i+1} c_{1}^{-1} \ldots c_{m-1} a_{i+1} c_{m-1}^{-1}$. We claim that $a_{i}^{*}$ is, again, $a_{i}$. As in case $1, a_{i}^{*}$ satisfies criteria 2 and 3 , so it only remains to show $a_{i}^{*} \in Z_{i}$. Again, we have

$$
\begin{aligned}
a_{i}^{*} / Z_{i} & =\left(c_{0} a_{i+1} c_{0}^{-1} c_{1} a_{i+1} c_{1}^{-1} \ldots c_{m-1} a_{i+1} c_{m-1}^{-1}\right) / Z_{i} \\
& =\left(c_{0} / Z_{i}\right)\left(a_{i+1} / Z_{i}\right)\left(c_{0}^{-1} / Z_{i}\right) \ldots\left(c_{m-1} / Z_{i}\right)\left(a_{i+1} / Z_{i}\right)\left(c_{m-1}^{-1} / Z_{i}\right) \\
& =\left(a_{i+1} / Z_{i}\right)^{m} \\
& =1 / Z_{i}
\end{aligned}
$$

since the exponent of any algebra in $\mathcal{V}$ divides $m$. So, again by minimality of $\pi_{i}$, we have that $a_{i}^{*}=a_{i}$, and so our polynomial has complexity at most $m$.

So, we have a sequence $\left(a_{i}\right)_{i=1}^{k}$ that walks down through the upper central series of $\mathbf{S}$, all the way down to $a_{1}$ which belongs to the center of $\mathbf{S}$. We can also walk $a_{1}$ down to some $a_{0}$ in the monolith via a polynomial $\pi_{0}$; the same proof suffices, as $Z_{1}$ is Abelian, so in particular its elements commute with every element of $M . a_{0} \in a^{S}$, as witnessed by the composition of each of the conjugate product polynomials $\pi_{i}$, which is itself a conjugate product polynomial. The complexity of the composition is bounded above by $m^{k}$. This completes the proof.

Now, we can complete the proof of Theorem 3.2. Let $T$ be the set of all conjugate product terms in the signature of $\mathcal{V}$ whose parameters are chosen from the distinct variables $u_{0}, \ldots, u_{m^{k}-1}$. Since the list of variables is finite, there are finitely many such terms. Now, let $\Psi(x, y)$ be the sentence

$$
\exists u_{0}, \ldots, u_{m^{k}-1}\left[\bigvee_{t \in T} t(y, \bar{u}) \approx x\right]
$$

Let $\Phi(x, y)$ be the normal closure formula from Theorem 4.1 that defines all atoms of congruence lattices of algebras in $\mathcal{V}$. Together, $\Phi(x, y)$ and $\Psi(x, y)$ witness that $\mathcal{V}_{\text {si }}$ has definable principal normal subgroups. 


\section{$5 \quad$ Future Research}

A number of natural followup questions to this result present themselves and beg to be investigated. For one, the original conjecture of McNulty and Wang is still open, as the current paper has only gone partway to solving it.

Problem 5.1. If $\mathcal{V}$ is a variety generated by a finite group $\mathbf{G}$, is it true that $\mathcal{V}_{\mathrm{si}}$ is finitely axiomatisable?

If a counterexample can be found to the conjecture, it is natural to want to know how far the finite axiomatisability can be taken. Is nilpotence the best we can do, or are there broader or perhaps unrelated classes of groups for which our result hold?

Problem 5.2. If $\mathcal{V}$ is a variety generated by some group $\mathbf{G}$, what conditions have to be met by $\mathbf{G}$ in order for $\mathcal{V}_{\mathrm{si}}$ to be finitely axiomatisable?

Universal algebraists will of course wish to extend this result to more general algebras besides just groups. We define an algebra to be a nonempty set $A$ along with finitary operations which define functions on $A$. Groups, rings, vector spaces and lattices all satsify this rather broad definition. Departing the isle of groups in favour of such general waters robs us of the concept of normal subgroups, so in universal algebra the attention is usually given to congruence relations.

A congruence relation on an algebra $\mathbf{A}$ is the relational kernel of some homomorphism. That is, given a homomorphism $h$, the associated congruence is the relational kernel $\{\langle a, b\rangle: h(a)=h(b)\}$. Congruences are also precisely the equivalence relations on $\mathbf{A}$ that are also subalgebras of $\mathbf{A}^{2}$. In groups, the equivalence classes of congruences are the left cosets of the normal subgroup that is the kernel of the homomorphism in question. The congruence class containing 1 is the kernel of the homomorphism. In algebras in general, we lack a unit element, and so the congruence relation as a kernel must replace the normal subgroup in most discussion.

The concept of the commutator in group theory has also been extended to a general commutator that exhibits similar properties in general algebras, at least in the case when the congruence lattices are modular, as is the case in groups. We refer readers to Freese and McKenzie's 1987 work [5] for an exhaustive discussion of commutator theory. This commutator enables an echo of concepts like Abelianness, nilpotence and solvability in varieties of algebras whose congruence lattices are modular. The algebraic commutator provides us with a lower central series. The concept of Abelian algebras similarly provides us with an upper central series, and as in groups, these two series have the same length.

Unfortunately, of the three characterisations of nilpotence we described earlier, the upper and lower central series are the only ones that extend to the algebraic commutator. The characterisation of a nilpotent group as one which is the product of its Sylow subgroups does not extend to general algebras, even in the otherwise well-behaved congruence modular varieties. What's worse, the notion of conjugate product polynomials is not so easily translated to algebras either, so the method of this paper does not seem to generalise beyond group theory. However, other methods might bear some fruit. The author has proved that the result extends to varieties of nilpotent algebras with the added hypothesis that the generating algebra is a product of algebras of prime power order. This proof should turn up in future publication. Nilpotent algebras in general remain a tougher nut to crack.

Problem 5.3. If $\mathcal{V}$ is a congruence modular variety generated by a finite nilpotent algebra $\mathbf{A}$, is it true that $\mathcal{V}_{\mathrm{si}}$ is finitely axiomatisable? 


\section{References}

[1] Kirby A. Baker and Ju Wang. 'Definable principal subcongruences'. Algebra Universalis 47 (2) (2002), 145-151.

[2] Garrett Birkhoff. 'On the structure of abstract algebras'. Mathematical Proceedings of the Cambridge Philosophical Society 31 (4) (1935), 433454.

[3] Garrett Birkhoff. 'Subdirect unions in universal algebra'. Bull. Amer. Math. Soc. 50 (1944), 764-768.

[4] David Steven Dummit and Richard M. Foote. Abstract Algebra (John Wiley and Sons, Inc., Hoboken, NJ, 2004), 3rd edn.

[5] Ralph Freese and Ralph McKenzie. Commutator theory for congruence modular varieties, London Mathematical Society Lecture Note Series, Volume 125 (Cambridge University Press, Cambridge, 1987).

[6] R. C. Lyndon. 'Two notes on nilpotent groups'. Proc. Amer. Math. Soc. 3 (1952), 579-583.

[7] H. Neumann. Varieties of Groups. Ergebnisse der Mathematik und ihrer Grenzgebiete. 2. Folge (Springer Berlin Heidelberg, 1967).

[8] Sheila Oates and M. B. Powell. 'Identical relations in finite groups'. J. Algebra 1 (1964), 11-39. 